\section{SENSITIVE QUANTIFICATION AND TRACKING OF THE ACTIVE COMPONENTS OF A CLONAL NEOANTIGEN T CELL (CNET) THERAPY: FROM MANUFACTURE TO PERIPHERAL CIRCULATION}

${ }^{1}$ Samra Turajlic*, ${ }^{2}$ Mariam Jamal-Hanjani, ${ }^{3}$ Andrew Furness, ${ }^{4}$ Ruth Plummer, ${ }^{5}$ Judith Cave, ${ }^{6}$ Fiona Thistlethwaite, ${ }^{7}$ Emma Leire, ${ }^{7}$ Jen Middleton, ${ }^{7}$ Eloise Williams, ${ }^{7}$ Amy Baker, ${ }^{7}$ Chloe Maine, ${ }^{7}$ Michael Epstein, ${ }^{7}$ Monica Sassi, ${ }^{7}$ Katy Newton, ${ }^{7}$ Michael Grant, ${ }^{7}$ Matilde Saggese, ${ }^{7}$ Sergio Quezada, ${ }^{2}$ Martin Forster. ${ }^{1}$ Royal Marsden NHS Foundation Trust, London, UK; ${ }^{2}$ UCL Cancer Institute, London, UK; ${ }^{3}$ Royal Marsden NHS Trust, London, UK; ${ }^{4}$ Newcastle Hospitals NHS Foundation Trust, London, UK; ${ }^{5}$ University Hospital Southampton, Southampton, UK; ${ }^{6}$ The Christie NHS Foundation Trust, Manchester, UK; ${ }^{7}$ Achilles Therapeutics UK Ltd, London, UK

Background Ex-vivo expanded tumour infiltrating lymphocytes (TIL) show promise in delivering durable responses among several solid tumour indications. However, characterising, quantifying and tracking the active component of TIL therapy remains challenging as the expansion process does not distinguish between tumour reactive and bystander T-cells. Achilles Therapeutics has developed ATL001, a patient-specific TILbased product, manufactured using the VELOS $^{\text {тм }}$ process that specifically targets clonal neoantigens present in all tumour cells within a patient. Two Phase I/IIa clinical trials of ATL001 are ongoing in patients with advanced Non-Small Cell Lung Cancer, CHIRON (NCT04032847), and metastatic or recurrent melanoma, THETIS (NCT03997474). Extensive product characterisation and immune-monitoring are performed through Achilles' manufacturing and translational science programme. This enables precise quantification and characterisation of the active component of this therapy Clonal Neoantigen $\mathrm{T}$ cells $(\mathrm{cNeT})$ - during manufacture and following patient administration, offering unique insight into the mechanism of action of ATL001 and aiding the development of next generation processes.

Methods ATL001 was manufactured using procured tumour and matched whole blood from 8 patients enrolled in the THETIS $(n=5)$ and CHIRON $(n=3)$ clinical trials. Following administration of ATL001, peripheral blood samples were collected up to week 6 . The active component of the product was detected via re-stimulation with clonal neoantigen peptide pools and evaluation of IFN- $\gamma$ and/or TNF- $\alpha$ production. Deconvolution of individual reactivities was achieved via ELISPOT assays. Immune reconstitution was evaluated by flow cytometry. cNeT expansion was evaluated by restimulation of isolated PBMCs with peptide pools and individual peptide reactivities (ELISPOT).

Results The median age was 57 (range 30 - 71) and 6/8 patients were male. The median number of previous lines of systemic anti-cancer treatment at the time of ATL001 dosing was 2.5 (range $1-5$ ). Proportion of $\mathrm{cNeT}$ in manufactured products ranged from $0.20 \%-77.43 \%$ (mean $26.78 \%$ ) and unique single peptide reactivities were observed in 7 of 8 products (range $0-28$, mean 8.6). Post-dosing, cNeTs were detected in $5 / 8$ patients and $\mathrm{cNeT}$ expansion was observed in $3 / 5$ patients.

Conclusions These data underscore our ability to sensitively detect, quantify and track the patient-specific cNeT component of ATL001 - during manufacture and post dosing. As the dataset matures, these metrics of detection and expansion will be correlated with product, clinical and genomic characteristics to determine variables associated with peripheral cNeT dynamics and clinical response.

Trial Registration NCT04032847, NCT03997474

\section{REFERENCES}

Ethics Approval The first 8 patients described have all been located within the UK and both trials (CHIRON and THETIS) have been approved by the UK MHRA (among other international bodies, e.g FDA). Additionally, these trials have been approved by local ethics boards at active sites within the UK. Patient's are fully informed by provided materials and investigators prior to consenting to enrol into either ATL001 trial.

http://dx.doi.org/10.1136/jitc-2021-SITC2021.543 